\title{
Effects of Viscous Dissipation on Unsteady Mixed Convection Heat Transfer from a Circular Cylinder for Parallel and Contra Flows
}

\author{
Abdullah A. A. A. Al-Rashed ${ }^{1}$, Nepal C. Roy ${ }^{2}$ and Md. Anwar Hossain ${ }^{2 *}$ \\ ${ }^{1}$ Department of Automotive and Marine Engineering Technology, College of Technological Studies, The Public Authority for \\ Applied Education and Training, Kuwait, ${ }^{2}$ Department of Mathematics, University of Dhaka, Dhaka, Bangladesh
}

Unsteady mixed convection heat transfer from a circular cylinder has been investigated in the presence of viscous dissipation. The isothermal horizontal cylinder is placed to the oncoming free stream whose direction is considered in the free convection flow (parallel flow) and opposite to it (contra flow). The system of dimensionless governing equations for unsteady, two-dimensional flow is reduced to a suitable form for integration, and the resulting equations are solved employing finite-difference method. For both parallel and

OPEN ACCESS

Edited by:

Dipankar Chatterjee,

CSIR-Central Mechanical Engineering

Research Institute, India

Reviewed by:

Sandip Sarkar,

Tata Global R\&D Division, India;

Indian Institute of Science, India

Amit Kumar Dhiman,

Indian Institute of Technology

Roorkee, India

*Correspondence:

Md. Anwar Hossain

anwar.cfd@gmail.com

Specialty section:

This article was submitted to Thermal and Mass Transport, a section of the journal Frontiers in Mechanical

Engineering

Received: 12 October 2016 Accepted: 16 February 2017

Published: 06 March 2017

Citation: Al-Rashed AAAA, Roy NC and Hossain MA (2017) Effects of Viscous

Dissipation on Unsteady Mixed Convection Heat Transfer from a Circular Cylinder for Parallel and Contra Flows.

Front. Mech. Eng. 3:2. doi: 10.3389/fmech.2017.00002 contra flows, the influences of the viscous dissipation on the Nusselt number are found to be strong; however, it has rather weak effect on the vorticity distribution. In the presence of the viscous dissipation, the isotherms are significantly changed while there is less effect on the streamlines. Under the same conditions, the size of the vortex for the contra flow is larger than that for parallel flow.

Keywords: viscous dissipation, mixed convection, circular cylinder, unsteady, parallel and contra flows

\section{INTRODUCTION}

Heat transfer from a circular cylinder has been the subject of research due to its occurrence in many industrial and engineering applications such as heat exchanger tubes, chimney stacks, cooling towers, measuring probes, and sensors (Sarkar et al., 2011), drying of different materials (textiles, veneer, paper, and film materials), cooling of glass, plastics, and industrial devices, from turbine blades to electronic circuits, anemometry, and chemical or radioactive contamination/purification (Juncu, 2004), nuclear reactors, hot wires, and steam pipes (Mahfouz, 2003). In some processes, it takes place only by free convection or forced convection. However, there are some processes where the forced and free convection are of comparable order, and then the process of heat transfer is known as combined convection or mixed convection. This paper investigates the heat-transfer characteristics of the combined free and forced convection from a horizontal circular cylinder, which is placed to a transverse flow whose direction is either in the free convection flow (parallel flow) or opposite to it (contra flow).

Dennis and Chang (1970) investigated the steady incompressible flow around a circular cylinder for a range of Reynolds numbers from 5 to 100. The dimensionless governing equations are solved employing finite-difference method, but in effect, it appears to be failed because of being very slow at higher Reynolds numbers. Results have been elucidated by the drag coefficient, the angle of separation, and the pressure and vorticity distributions over the cylinder surface. Almost a decade later, Fornberg (1980) considered the same problem and carried out an efficient numerical method based on Newton's method, which can provide solutions beyond a Reynolds number of 100 . Numerical solutions showed that just before a Reynolds number of 300 , the wake region is reduced or elongated when the vorticity regenerates from the end of the wake region. 
Mahfouz (2003) considered the transient free convection from an isothermal cylinder placed horizontally in a micropolar fluid. The local and average Nusselt numbers and the flow and thermal fields have been discussed for different values of the controlling parameters. The natural convection heat transfer from an isothermal horizontal cylinder of elliptic cross section has been examined by Cheng (2006). The viscosity of the fluid is assumed to vary proportional to an inverse linear function of temperature and a suitable coordinate transformation, and the cubic spline collocation method was used. Moreover, Juncu (2008) investigated the transient heat transfer from an elliptic cylinder placed in a free stream fluid, and the temperature of the cylinder varies in time.

A good work on the forced convection heat transfer from a circular cylinder has been done by Kurdyumov and Fernández (1998). Results are illustrated in terms of the Nusselt number for small values of Reynolds number. Nguyen et al. (1996) studied the heat transfer from a rotating circular cylinder. It was embedded in a spatially uniform and time-dependent convective environment under the effect of buoyancy force. The equations of the vorticity, stream function, and energy were solved using a hybrid spectral scheme. The Reynolds number, Grashof number, rotational speed, and the gravity direction had considerable influence on the heat-transfer rate. The effects of Prandtl number and Richardson number on the wake dynamics and heat transfer past a circular cylinder in crossflow have been examined by Sarkar et al. (2011). The effects of the Reynolds number and Prandtl number have been illustrated in terms of the local and average Nusselt.

In the nineteenth century, Badr and his coworkers published a series of papers (Badr, 1983, 1984; Badr and Dennis, 1985; Badr et al., 1990) on the flow and heat transfer around a cylinder. Badr (1983) examined the laminar mixed convection from a horizontal cylinder placed in the free stream, which is directed to horizontal and perpendicular to the cylinder axis. Results are presented in terms of vorticity, pressure, temperature, and local Nusselt number around the surface. The author also considered the same problem taking the forced flow directed either vertically upward (parallel flow) or vertically downward (contra flow). The vorticity and Nusselt number distributions are illustrated with the change of the Grashof number and the Reynolds number for both parallel and contra flows. Mahfouz and Badr (2000) numerically demonstrated the characteristics of the flow in the wake of a circular cylinder, which was kept in a cross-stream and made rotational oscillation about its own axis.

Biswas and Sarkar (2009) examined the effects of thermal buoyancy on vortex shedding behind a circular cylinder, which is placed in a crossflow at low Reynolds numbers. The dimensionless governing equations have been solved using streamline upwind Petrov-Galerkin (SUPG) based finite element method. Sarkar et al. (2010) studied the flow and heat-transfer characteristics for mixed convection flow past two tandem square cylinders which were kept in a uniform upward flow at a Reynolds number of 100. Numerical solutions of the problem are obtained using the aforementioned SUPG based finite element method, and the results have been demonstrated for various Richardson numbers taking into consideration the influences of aiding and opposing buoyancy.
A long time ago, Nakai and Okazaki (1975) examined the heat-transfer characteristics from a horizontal infinite fine wire by mixed convections for parallel, contrary, and crossflows. The problem was analyzed theoretically considering the dominant behavior of one of the forced or free convection to the other. They also conducted an experiment and found a good agreement with the analytical solutions. Gebhart (1962) performed boundary layer analysis in order to comprehend the influence of the viscous dissipation for vertical surfaces under different thermal conditions. The situations where viscous dissipation is important have been illustrated elaborately. He concluded that the magnitude of the viscous dissipation effect is dependent on a dissipation number; however, it cannot be expressed in terms of the Grashof or the Prandtl number. Abu-Hijleh et al. (1998) studied the effect of viscous dissipation on the local entropy generation due to natural convection from a heated horizontal isothermal cylinder. They mentioned that the comprehensive knowledge about the overall natural convective heat transfer around circular cylinders is important for the development of heat exchangers, hot water and steam pipes, heaters, refrigerators, and electrical conductors. Recently, Chhabra et al. (2007) investigated the effects of viscous dissipation on the heat transfer between banks of long cylinders and power law fluids.

From the above literature survey, it can be concluded that a very few studies have considered the mixed convection heat transfer from a circular cylinder for both parallel and contra flows. Moreover, the previous studies except Abu-Hijleh et al. (1998) and Chhabra et al. (2007) have neglected the influences of viscous dissipation, but it is always not realistic. For this reason, the objective of this study is to investigate the unsteady mixed convection heat transfer from a circular cylinder for both parallel and contra flows in the presence of viscous dissipation. The system of governing equations is transformed into a suitable form of integration, and the resulting equations are solved using finite-difference method. Numerical results are presented in terms of the Nusselt number and the vorticity distribution as well as the streamlines and the isotherms.

\section{MATHEMATICAL FORMULATION}

We consider a horizontal circular cylinder of radius $a$ in a uniform free stream of velocity $U_{\infty}$ and temperature $T_{\infty}$ while the surface of the cylinder is kept at constant temperature $T_{s}$. The flow direction of the free stream has been taken in two ways: vertically upward and vertically downward. We also assume that the temperature difference $\left(T_{s}-T_{\infty}\right)$ between the cylinder surface and the ambient fluid has a negligible effect on the fluid properties; however, it can significantly affects the buoyancy forces in the momentum equation. Moreover, the cylinder is presumed to be long enough that produces negligible end effects. In this manner, we can consider the flow field to be two-dimensional. Therefore, the governing equations can be expressed in terms of vorticity, stream function, and energy equations as

$$
\begin{aligned}
\frac{\partial \omega^{\star}}{\partial t^{\star}}+u^{\star} \frac{\partial \omega^{\star}}{\partial r^{\star}}+\frac{v^{\star}}{r^{\star}} \frac{\partial \omega^{\star}}{\partial r^{\star}} & =v \nabla^{2} \omega^{\star} \\
& \pm \frac{1}{\rho}\left(\frac{\partial F_{\theta}}{\partial r^{\star}}-\frac{1}{r^{\star}} \frac{\partial F_{r^{\star}}}{\partial \theta}+\frac{F_{\theta}}{r^{\star}}\right)
\end{aligned}
$$




$$
\begin{gathered}
\omega^{\star}=\nabla^{2} \psi^{\star}, \\
\frac{\partial T}{\partial t^{\star}}+u^{\star} \frac{\partial T}{\partial r^{\star}}+\frac{v^{\star}}{r^{\star}} \frac{\partial T}{\partial \theta}=\frac{\kappa}{\rho c} \nabla^{2} T+\frac{\nu}{c} \Phi^{\star},
\end{gathered}
$$

where

$$
\nabla^{2}=\frac{\partial^{2}}{\partial r^{\star 2}}+\frac{1}{r^{\star}} \frac{\partial}{\partial r^{\star}}+\frac{1}{r^{\star 2}} \frac{\partial^{2}}{\partial \theta^{2}},
$$

and $\Phi^{\star}$ is the viscous dissipation term and given by

$$
\begin{aligned}
\Phi^{\star}= & \left(\frac{1}{r^{\star}} \frac{\partial u^{\star}}{\partial \theta}+\frac{\partial v^{\star}}{\partial r^{\star}}-\frac{v^{\star}}{r^{\star}}\right)^{2} \\
& +2\left\{\left(\frac{\partial u^{\star}}{\partial r^{\star}}\right)^{2}+\frac{1}{r^{\star} 2}\left(u^{\star}+\frac{\partial v^{\star}}{\partial \theta}\right)^{2}\right\} .
\end{aligned}
$$

In the above equations, $t^{*}$ is the time, $u^{\star}$ and $v^{*}$ are the velocities in the $r^{*}$ and $\theta$ directions, $T$ is the temperature, $\rho$ is the density, $v$ is the kinematic viscosity, $\kappa$ is the thermal conductivity, and $c$ is the specific heat. Moreover, $F_{r^{\star}}$ and $F_{\theta}$ are the radial and transverse components of the body force and defined as

$$
F_{r^{\star}}=-\rho g \beta\left(T-T_{\infty}\right) \cos \theta \quad \text { and } \quad F_{\theta}=\rho g \beta\left(T-T_{\infty}\right) \sin \theta,
$$

where $g$ is the gravitational acceleration, $\beta$ is the coefficient of thermal expansion, and $T_{\infty}$ is the fluid temperature far away from the cylinder. The vorticity $\omega$ and stream function $\psi$ are related to the velocity field by

$$
\omega=-\frac{1}{r^{\star}}\left(r^{\star} \frac{\partial v^{\star}}{\partial r^{\star}}+v^{\star}-\frac{\partial u^{\star}}{\partial \theta}\right)
$$

and

$$
u^{\star}=\frac{1}{r^{\star}} \frac{\partial \psi^{\star}}{\partial \theta}, \quad v^{\star}=-\frac{\partial \psi^{\star}}{\partial r^{\star}} .
$$

The associated boundary conditions are

$$
u^{\star}=v^{\star}=0, T=T_{s} \text { at } r=a,
$$

$$
\begin{aligned}
u^{\star} & =U_{\infty} \cos \theta, \quad v^{\star}=-U_{\infty} \sin \theta, \quad \omega^{\star}=0, \quad \text { and } \\
T & =T_{\infty} \quad \text { as } r \rightarrow \infty .
\end{aligned}
$$

We now introduce the following dimensionless variables

$$
\begin{gathered}
u=\frac{u^{\star}}{U_{\infty}}, \quad v=\frac{v^{\star}}{U_{\infty}}, \quad r=\frac{r^{\star}}{a}, \quad t=\frac{U_{\infty} t^{\star}}{a}, \\
\psi=\frac{\psi^{\star}}{a U_{\infty}}, \quad \omega=\frac{a \omega^{\star}}{U_{\infty}}, \quad \text { and } \quad \phi=\frac{T-T_{\infty}}{T_{s}-T_{\infty}},
\end{gathered}
$$

where $U_{\infty}$ is the velocity of the uniform stream.

Thus the system of Eqs 1-3 reduces to

$$
\begin{gathered}
\frac{\partial \omega}{\partial t}+u \frac{\partial \omega}{\partial r}+\frac{v}{r} \frac{\partial \omega}{\partial \theta}=\frac{2}{\operatorname{Re}} \nabla^{2} \omega \pm \frac{\mathrm{Gr}}{2 \operatorname{Re}^{2}}\left(\sin \theta \frac{\partial \phi}{\partial r}+\cos \theta \frac{\partial \phi}{\partial \theta}\right) \\
\omega=\nabla^{2} \psi \\
\frac{\partial \phi}{\partial t}+u \frac{\partial \phi}{\partial r}+\frac{v}{r} \frac{\partial \phi}{\partial Y}=\frac{2}{\operatorname{Pr} \operatorname{Re}} \nabla^{2} \phi+\operatorname{Ec} \Phi
\end{gathered}
$$

where

$$
\begin{aligned}
\nabla^{2} & =\frac{\partial^{2}}{\partial r^{2}}+\frac{1}{r} \frac{\partial}{\partial r}+\frac{1}{r^{2}} \frac{\partial^{2}}{\partial \theta^{2}}, \\
\Phi & =\left(\frac{1}{r} \frac{\partial u}{\partial \theta}+\frac{\partial v}{\partial r}-\frac{v}{r}\right)^{2}+2\left\{\left(\frac{\partial u}{\partial r}\right)^{2}+\frac{1}{r^{2}}\left(u+\frac{\partial v}{\partial \theta}\right)^{2}\right\} .
\end{aligned}
$$

Also the velocity components $u$ and $v$ used in the above equations are given by

$$
u=\frac{1}{r} \frac{\partial \psi}{\partial \theta}, \quad v=-\frac{\partial \psi}{\partial r} .
$$

In Eqs 10 and $12, \mathrm{Gr}=g \beta(2 a)^{3}\left(T_{s}-T_{\infty}\right) / v^{2}$ is the Grashof number, $\operatorname{Re}=2 a U_{\infty} / \nu$ is the Reynolds number, $\operatorname{Pr}=\mu c / \kappa$ is the Prandtl number, and $\mathrm{Ec}=v U_{\infty} / c a\left(T_{s}-T_{\infty}\right)$ is the Eckert number.

The boundary conditions take the form

$$
\begin{gathered}
u=v=0, \quad \phi=1 \quad \text { at } r=1, \\
u=\cos \theta, \quad v=-\sin \theta, \quad \omega=0, \quad \text { and } \phi=0 \quad \text { as } r \rightarrow \infty .
\end{gathered}
$$

Finally, we transform the polar coordinates $(r, \theta)$ into $(\xi, \theta)$ using the relation $\xi=\ln r$ so that Eqs 10-12 become

$$
\begin{aligned}
e^{2 \xi} \frac{\partial \omega}{\partial t}+U \frac{\partial \omega}{\partial \theta}+V \frac{\partial \omega}{\partial \xi}= & \frac{2}{\operatorname{Re}}\left(\frac{\partial^{2} \omega}{\partial \theta^{2}}+\frac{\partial^{2} \omega}{\partial \xi^{2}}\right) \\
& \pm \frac{\mathrm{Gr}}{2 \operatorname{Re}^{2}} e^{\xi}\left(\sin \theta \frac{\partial \phi}{\partial \theta}+\cos \theta \frac{\partial \phi}{\partial \xi}\right) \\
e^{2 \xi} \omega= & \frac{\partial^{2} \psi}{\partial \theta^{2}}+\frac{\partial^{2} \psi}{\partial \xi^{2}} \\
e^{2 \xi} \frac{\partial \phi}{\partial \tau}+U \frac{\partial \phi}{\partial \theta}+V \frac{\partial \phi}{\partial \xi} & =\frac{2}{\operatorname{Pr} \operatorname{Re}} \nabla^{2} \phi+e^{-2 \xi} \mathrm{Ec} \Phi
\end{aligned}
$$

where we have used the relations

$$
V=\frac{\partial \psi}{\partial \theta}, \quad U=-\frac{\partial \psi}{\partial \xi},
$$

$\Phi=\left(\frac{\partial U}{\partial \theta}+\frac{\partial V}{\partial \xi}-2 V\right)^{2}+2\left\{\left(\frac{\partial U}{\partial \xi}\right)^{2}+\left(U+\frac{\partial V}{\partial \theta}\right)^{2}\right\}$.

The corresponding boundary conditions are

$$
\begin{aligned}
\psi & =\frac{\partial \psi}{\partial \xi}=0 \quad \text { and } \phi=1 \quad \text { at } \xi=0, \\
\frac{\partial \psi}{\partial \theta} & =e^{\xi} \cos \theta, \quad \frac{\partial \psi}{\partial \xi}=e^{\xi} \sin \theta, \quad \omega=0, \quad \phi=0 \quad \text { as } \xi \rightarrow \infty .
\end{aligned}
$$

From physical point of view, the local Nusselt number, $\mathrm{Nu}$, and the average skin friction, $\bar{C}_{f}$, and Nusselt number, $\overline{\mathrm{Nu}}$, are of great importance. These are defined as

$$
\mathrm{Nu}=\frac{a h}{k}, \quad \bar{C}_{f}=\frac{a \bar{\tau}_{w}}{\mu U_{\infty}}, \quad \text { and } \quad \overline{\mathrm{Nu}}=\frac{a \bar{h}}{k},
$$

where $h$ and $\bar{h}$ are, respectively, the local and overall heat-transfer coefficients, which are given by

$$
\begin{aligned}
\bar{\tau}_{w} & =\frac{\mu}{\pi} \int_{0}^{\pi}\left(\frac{\partial u^{\star}}{\partial r^{\star}}\right)_{r^{\star}=a} d \theta, \quad h=\frac{\kappa}{T_{s}-T_{\infty}}\left(\frac{\partial T}{\partial r^{\star}}\right)_{r^{\star}=a}, \text { and } \\
\bar{h} & =\frac{1}{2 \pi} \int_{0}^{2 \pi} h d \theta .
\end{aligned}
$$




\section{NUMERICAL METHOD}

In this study, we have solved the dimensionless governing Eqs $16-18$ in the modified coordinates $(\xi, \theta)$ using finite-difference method. In particular, we use SOR method with residual tolerance of order $10^{-6}$ for the stream function defined in Eq. 17. Taking $\xi_{\max }=4.0$, we consider the uniform meshes of sizes $\Delta \theta=2 \pi /(m-1)$ and $\Delta \xi=\xi_{\max } /(n-1)$, where $m=257$ and $n=129$ are the number of intervals along the $\theta$ and $\xi$ directions. The relaxation parameter, say " $\delta$ " is determined from the relation (Roache, 1998):

$$
\delta=2\left(\frac{1-\sqrt{1-\varepsilon}}{\varepsilon}\right)
$$

where

$$
\varepsilon=\frac{1}{4}\left[\cos \left(\frac{\pi}{m-1}\right)+\cos \left(\frac{\pi}{n-1}\right)\right]^{2} .
$$

Having found the stream function, we calculate the velocity components using Eq. 19 at each time step. Then the alternate direct implicit (ADI) method is used for the transient vorticity transport and energy Eqs 16 and 18. In this manner, using the values of $\omega$ and $\phi$ at any time step, we compute their values at the next time step from Eqs 16 and 18. It is noted that the transient and diffusion terms in the ADI method are discretized using forwardtime central-space technique. Moreover, we use second upwind differencing technique for the convective terms. Although ADI method is unconditionally stable, a grid independency test has been performed taking different number of mesh points, and the results are presented in Table 1. Here, we calculate the percentage error (\%) for a variable $\Omega$ using two computed values with different number of grid points, which is given by

$$
\text { Error }(\%)=\left|\frac{\Omega\left(m_{k+1} \times n_{k+1}\right)-\Omega\left(m_{k} \times n_{k}\right)}{\Omega\left(m_{k} \times n_{k}\right)}\right| \times 100
$$

where $m_{k}=2^{k}+1$ and $n_{k}=2^{k-1}+1$ denote the number of grids in $\theta$ and $\xi$ directions, respectively. In order to justify the grid dependency test, the average Nusselt number is calculated considering different number of mesh points. When we determine the percentage error of $\Omega$ for $65 \times 33$ grids, the value of $\Omega$ for $33 \times 17$ grids is taken as a reference value. It is clear that the maximum error in the average Nusselt number calculated for mesh size $257 \times 129$ and $513 \times 257$ becomes less than $2 \%$. Accordingly, we choose a grid of size $257 \times 129$ as a standard grid for the entire computation. Above all, the reduction in relative error validates the grid independence of the solution.

TABLE 1 | Comparison of average skin friction and Nusselt number for parallel flow at $\tau=10$ when $\operatorname{Pr}=0.7, \operatorname{Re}=20, \mathrm{Ec}=0.01, \mathrm{Gr}=100$, and $\Delta \tau=10^{-4}$.

\begin{tabular}{lcccr}
\hline Grids & $\overline{\boldsymbol{C}}_{\boldsymbol{f}}$ & Error (\%) & $\overline{\mathbf{N u}}$ & Error (\%) \\
\hline $65 \times 33$ & -3.17484 & 5.1 & 1.42745 & 14.8 \\
$129 \times 65$ & -3.07374 & 3.2 & 1.35318 & 5.5 \\
$257 \times 129$ & -3.01313 & 1.97 & 1.33385 & 1.5 \\
$513 \times 257$ & -2.98728 & 0.9 & 1.32645 & 0.6
\end{tabular}

\section{RESULTS AND DISCUSSION}

In Figure 1, a comparison is made of the average Nusselt number obtained by the present method and Badr (1983). As mentioned by Badr (1983), the cylinder was heated instantaneously to the constant surface temperature $T_{s}$ after a certain time. So the result indicates the decrease of average Nusselt after a certain time. However, the present study evaluates the time from 0 . Thus, the initial time taken by Badr (1983) has been transformed into 0 for the validation purpose. It is evident from the result that the present method gives a good agreement with Badr (1983). Moreover, Figure 2 shows the comparison of vorticity distribution obtained by the present method and Badr (1983). In this case, there is found an excellent agreement between the present method and Badr (1983).

The transient development of streamlines and isotherms for the parallel flow is demonstrated in Figures $3 \mathrm{~A}-\mathrm{C}$ and $\mathbf{4 A - C}$, respectively. As time goes by, the size of the vortex and the momentum boundary layer gradually increase. On the other hand, Figure $4 \mathrm{~A}-\mathrm{C}$ shows that the temperature distribution is elongated with time in the downstream region. Moreover, it becomes thick at the rear stagnation point $(\theta=180)$. The reasons for increasing the vortex and the momentum boundary layer

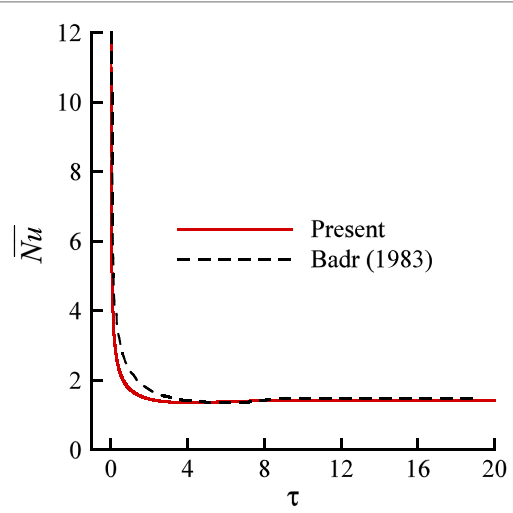

FIGURE 1 | A comparison with the result obtained by Badr (1983) for $\operatorname{Pr}=0.73, \operatorname{Re}=5$, and $\mathrm{Gr}=100$

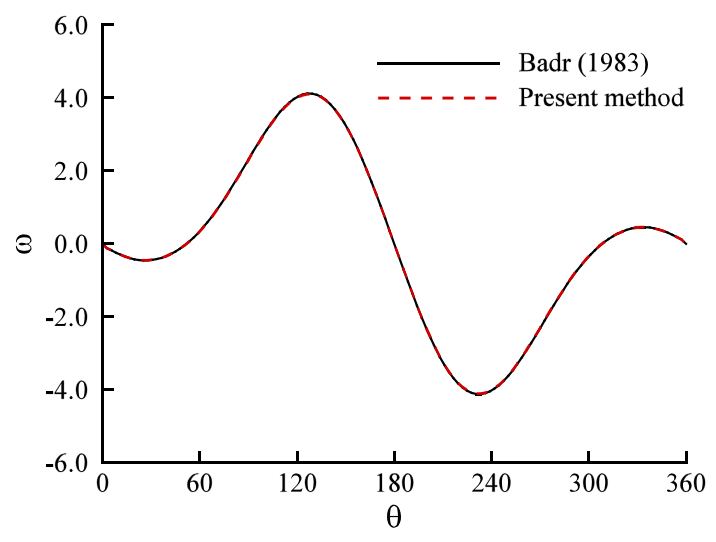

FIGURE 2 | Vorticity distribution obtained by the present method and Badr (1983) when $\operatorname{Pr}=0.7, \operatorname{Re}=20$, and $\mathrm{Gr}=100$. 


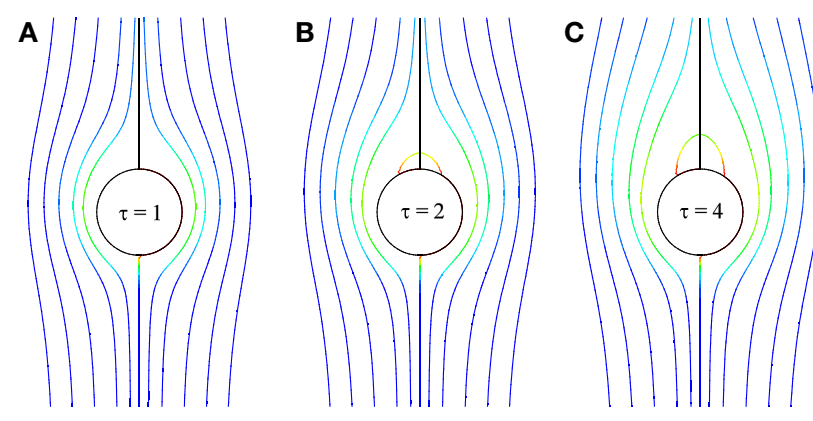

FIGURE 3 | Transient development of streamlines for the parallel flow for $\operatorname{Pr}=0.7, \operatorname{Re}=20, \mathrm{Ec}=0.01$, and $\mathrm{Gr}=100$.
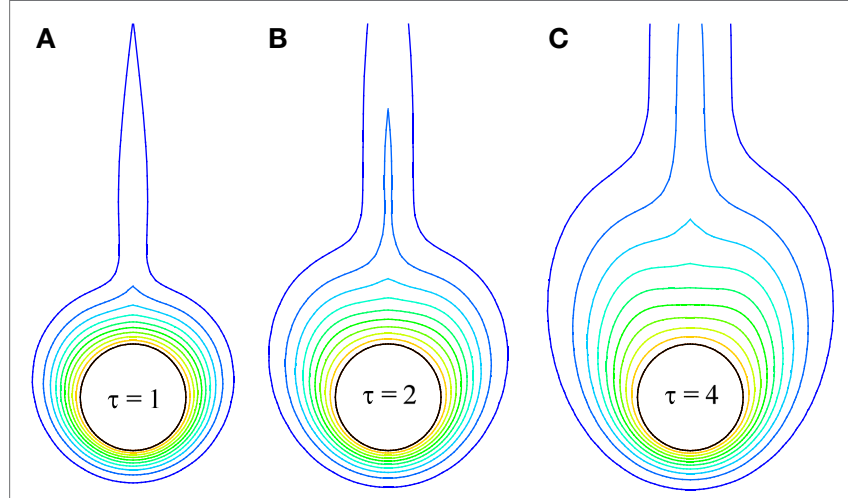

FIGURE 4 | Transient development of isotherms for the parallel flow for $\operatorname{Pr}=0.7, \operatorname{Re}=20, E c=0.01$, and $\mathrm{Gr}=100$.

and thickening the temperature distribution are attributed to the convection of fluid and heat along the direction of the free stream.

Figures 5A-C and 6A-C depict the transient development of streamlines and isotherms, respectively, for the contra flow. It is found that there occurs vortex within a short period of time. With increase of time, it significantly increases. This is due to the fact that the fluid erupts against the buoyancy force which induces negative pressure at the rear stagnation point. It is evident from Figure $\mathbf{6 A - C}$ that the temperature distribution is drastically elongated with time in the downstream region. Also the thickness of the temperature distribution increases at the rear stagnation point $(\theta=180)$, which helps to increase the size of the vortex. Since the hot fluid near the circular cylinder is transferred by the free stream, hence the temperature distribution is extended in the downstream region.

The effects of the Eckert number on the local Nusselt number for parallel and contra flows are shown in Figures 7A,B, respectively. When $\mathrm{Ec}=0.0$, the Nusselt number for parallel flow gradually increases along the surface of the cylinder from the front stagnation point $\theta=0$ and gets a maximum at the rear stagnation point $\theta=\pi$. In the presence of viscous dissipation, that is, when $\mathrm{Ec} \neq 0$, the Nusselt number for parallel flow demonstrates the non-monotonous behavior in the interval $0 \leq \theta \leq 2 \pi / 3$ and then it increases with $\theta$ up to $\theta=\pi$. The similar characteristics are observed when the angular distance changes from $2 \pi$ to $\pi$. But for contra flow, the Nusselt number corresponding to any of the

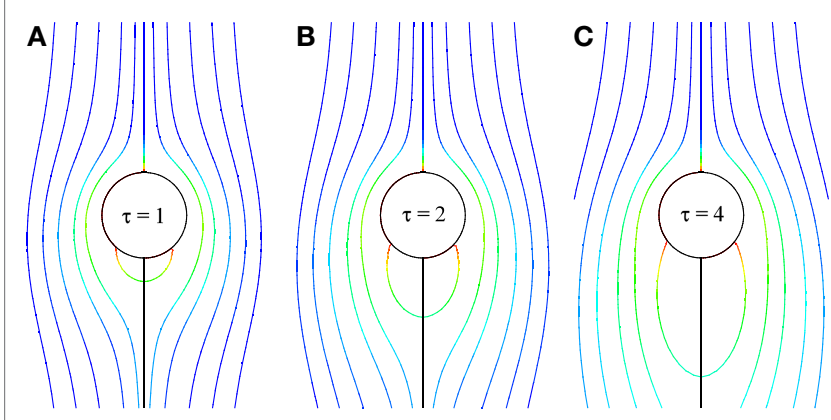

FIGURE 5 | Transient development of streamlines for the contra flow for $\operatorname{Pr}=0.7, \operatorname{Re}=20, \mathrm{Ec}=0.01$, and $\mathrm{Gr}=100$.
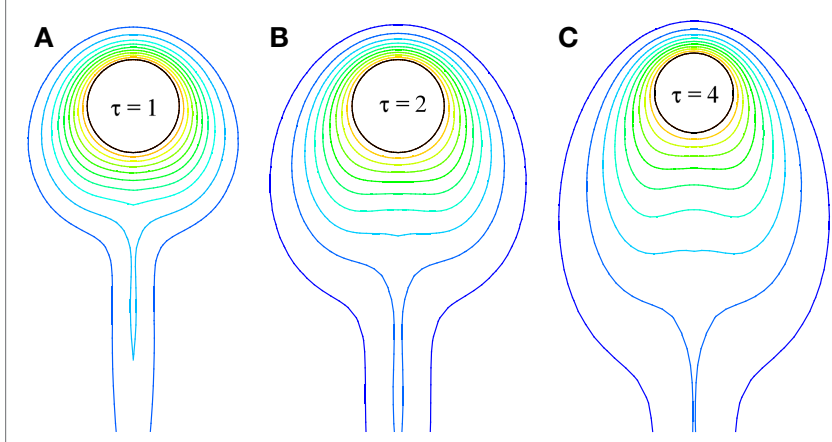

FIGURE 6 | Transient development of isotherms for the contra flow for $\operatorname{Pr}=0.7, \operatorname{Re}=20, \mathrm{Ec}=0.01$, and $\mathrm{Gr}=100$.

Eckert number changes almost in the same manner of parallel flow for $\mathrm{Ec}=0.0$. In both cases, the Eckert number has strong effect on the Nusselt number and, it decreases with increasing values of the Eckert number. In particular, the Nusselt number relating to $\mathrm{Ec} \neq 0$ has distinct characteristics from that for $\mathrm{Ec}=0.0$ in the interval $0 \leq \theta \leq 2 \pi / 3$.

The effects of the Eckert number on the vorticity distribution for parallel and contra flows are demonstrated in Figures 8A,B, respectively. It is found from Figure $\mathbf{8 A}$ that whatever be the value of Ec the vorticity first increases in the interval $(0,2 \pi / 3)$ and then decreases up to the point $\theta=\pi$. However, it varies in a reverse way as $\theta$ changes from $2 \pi$ to $\pi$. There is seen weak effect of the Eckert number on the vorticity distribution. In the case of parallel flow, the maximum value of the Nusselt number occurred at $\theta=2 \pi / 3$ increases, and its minimum value at $\theta=4 \pi / 3$ decreases as the value of Ec increases. The similar characteristics are observed for contra flow. But the effect of the Eckert number on the vorticity distribution for contra flow seems to be higher than that for parallel flow.

Figures 9A,B illustrate the variations of the steady state streamlines and isotherms for parallel and contra flows. Results indicate that the momentum boundary layer and the thermal boundary layer become thinner for parallel flow compared to those for contra flow. The reason is that in the case of parallel flow the forced convection aids the buoyancy force owing to their same direction which accordingly accelerates the fluid flow. Moreover, the points of separation for parallel flow are delayed in comparison with contra flow. This is due to the fact that in the case of parallel flow, 

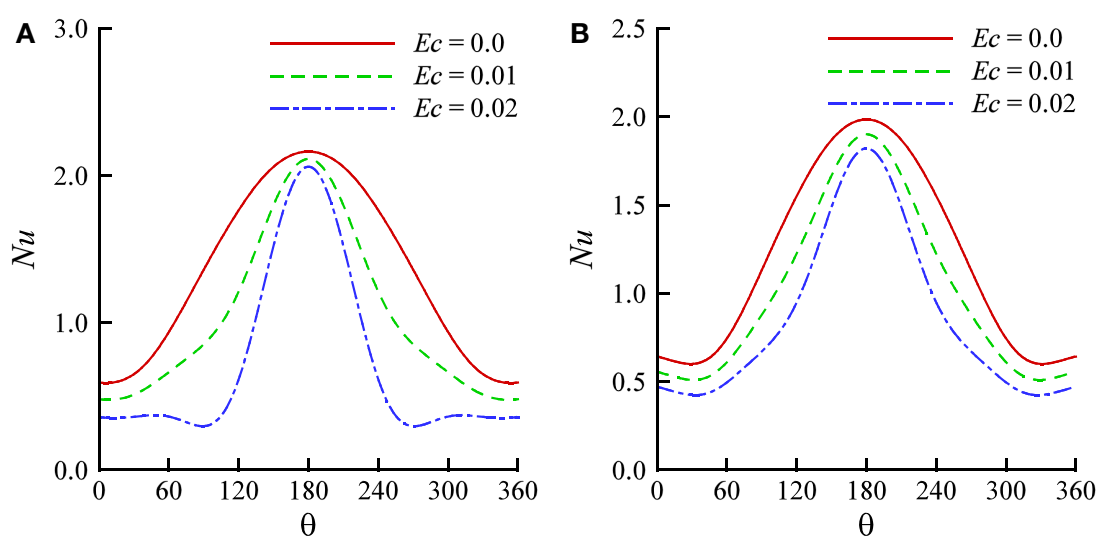

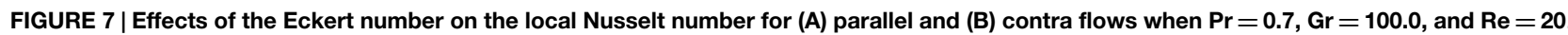
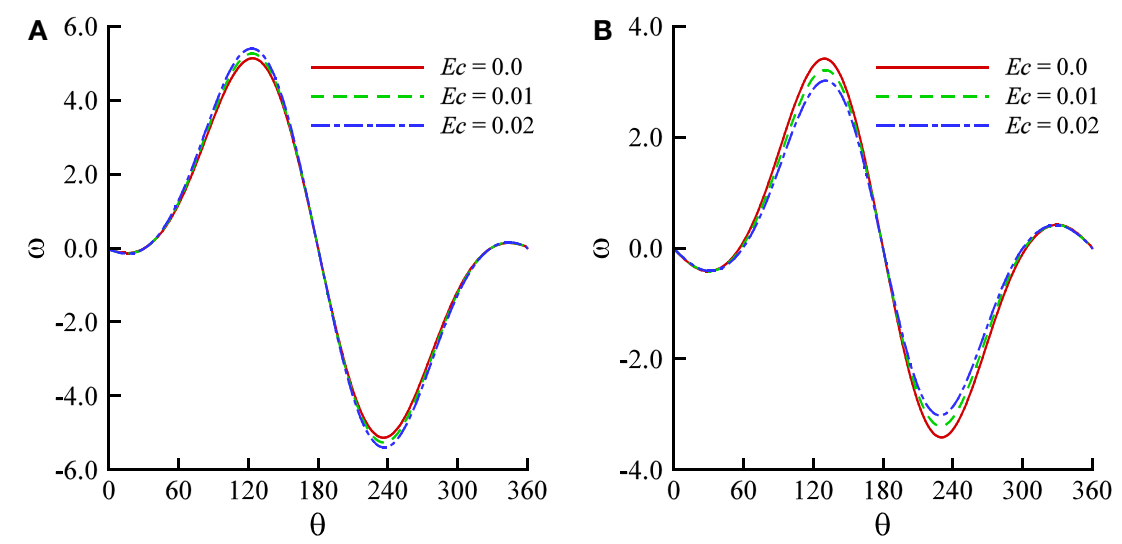

FIGURE 8 | Effects of the Eckert number on the vorticity distribution for (A) parallel and (B) contra flows when $\operatorname{Pr}=0.7, \mathrm{Gr}=100.0$, and $\mathrm{Re}=20$
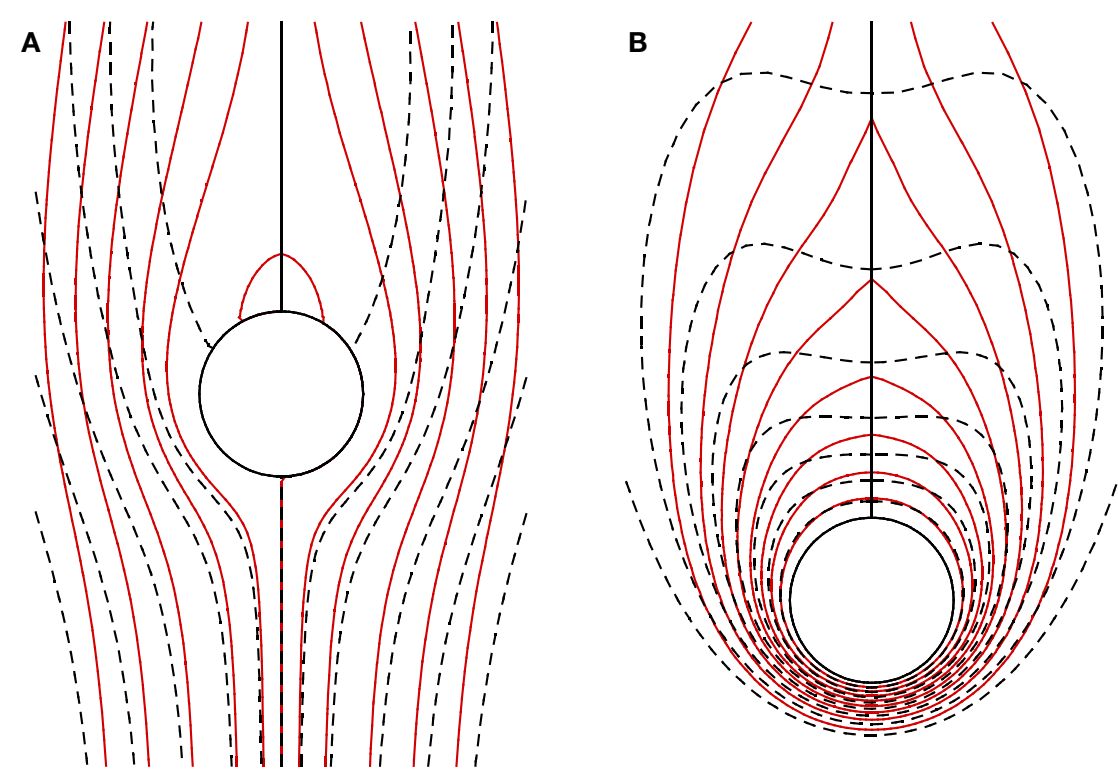

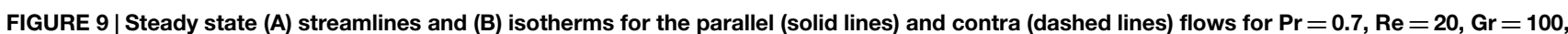
and $\mathrm{Ec}=0.01$. 
the forced convection assists the free convection flow resulting in increasing the fluid flow, which inhibits the flow field to be separated from the surface of the cylinder.

The influences of the Eckert number on the streamlines and isotherms for the parallel flow are shown in Figures 10A,B, respectively. It is clear from Figure 10A that there is weak effect of the Eckert number on the fluid flow, and it thickens the momentum boundary layer at a small amount. It is because heat is dissipated around the circular cylinder, which is then transferred in the direction of free convection flow. However, the Eckert number has tremendous effect on the temperature distribution. Due to dissipation of heat, the temperature distribution erupts not only in the downstream region but also in the radial direction. For this reason, the thermal boundary layer increases with Eckert number.

Figures 11A,B exhibit the effects of the Eckert number on the streamlines and isotherms, respectively. Figure 11B shows that heat is dissipated around the cylinder in a wide region with the
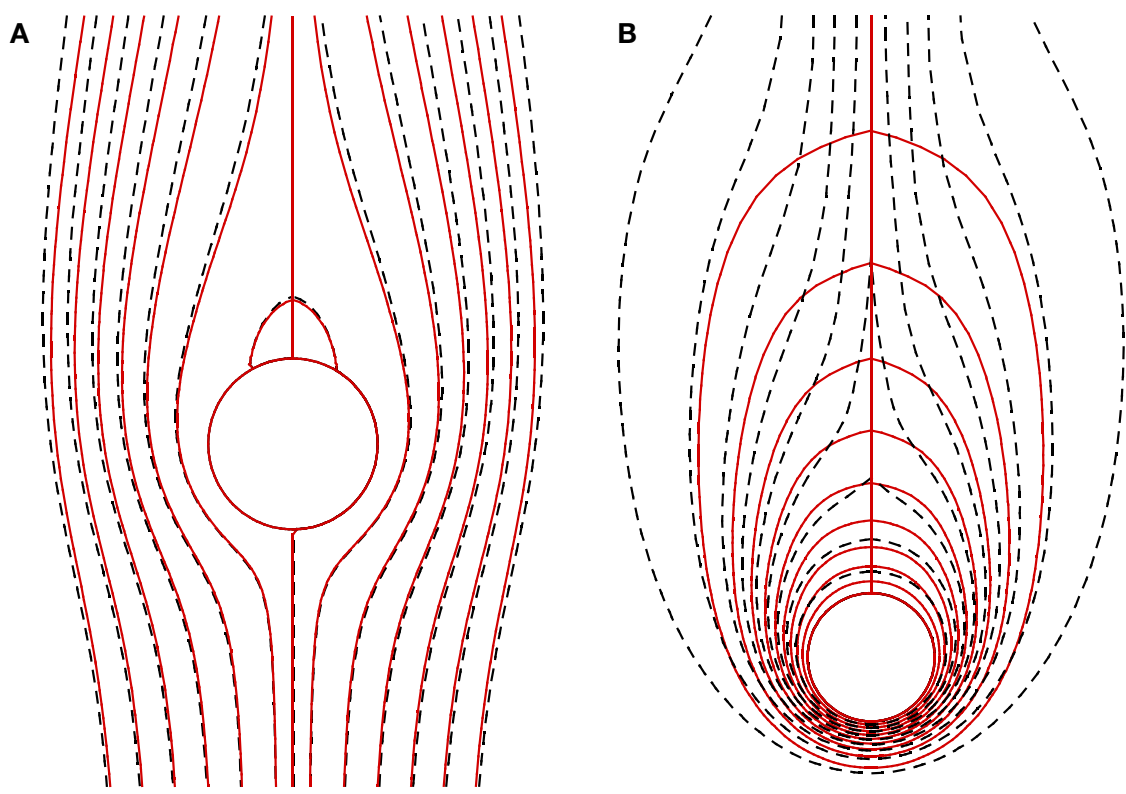

FIGURE 10 | Steady state (A) streamlines and (B) isotherms for the parallel flow when $\operatorname{Pr}=0.7, \operatorname{Re}=20, \mathrm{Gr}=100, \mathrm{Ec}=0.0$ (solid lines), and $\mathrm{Ec}=0.02$ (dashed lines)
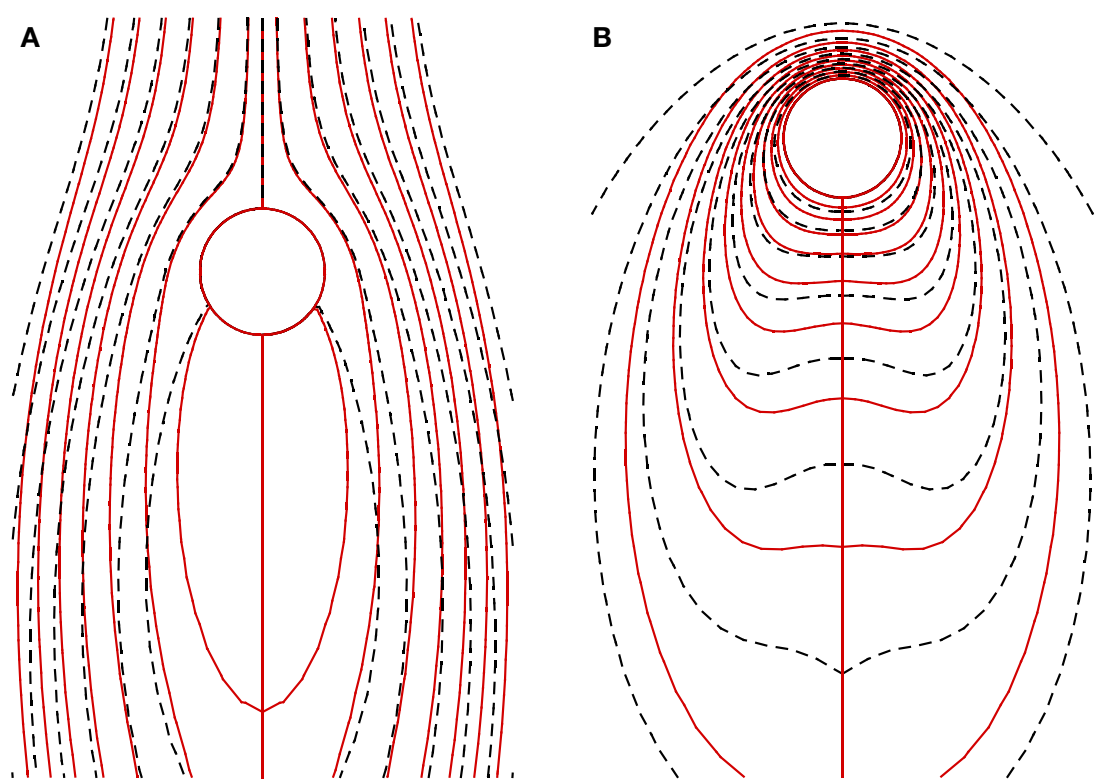

FIGURE 11 | Steady state (A) streamlines and (B) isotherms for the contra flow when $\operatorname{Pr}=0.7, \operatorname{Re}=20, \mathrm{Gr}=100, \mathrm{Ec}=0.0$ (solid lines), and Ec $=0.02$ (dashed lines) 
presence heat dissipation. Also the temperature distribution is significantly elongated owing to the combined effect of buoyancy and forced convection. That is why the thermal boundary layer considerably increases with the dissipation of heat. Besides it is evident from Figure 11A that there is negligible effect of the Eckert number on the streamlines, especially in the upstream region. However, the size of the vortex increases with the dissipation of heat, although the point of separation remains almost same. In comparison with $\mathrm{Ec}=0.0$, the cause of increase of the momentum and thermal boundary layer for $\mathrm{Ec}=0.02$ is the dissipation of heat, which results in the eruption of the fluid against the buoyancy force owing to density differences.

\section{CONCLUSION}

The unsteady mixed convection heat transfer from a circular cylinder is investigated in the presence of heat dissipation. Two

\section{REFERENCES}

Abu-Hijleh, B. A. /K., Jadallah, I. N., and Nada, E. A. (1998). Entropy generation due to natural convection from a horizontal isothermal cylinder in oil. Int. Commun. Heat Mass Transf. 25, 1135-1143. doi:10.1016/S0735-1933(98)00104-3

Badr, H. M. (1983). A theoretical study of laminar mixed convection from a horizontal cylinder in cross stream. Int. J. Heat Mass Transf. 26, 639-653. doi:10.1016/0017-9310(83)90014-5

Badr, H. M. (1984). Laminar combined convection from a horizontal cylinder-parallel and contra flow regimes. Int. J. Heat Mass Transf. 27, 15-27. doi:10.1016/0017-9310(84)90233-3

Badr, H. M., Coutanceau, M., Dennis, S. C. R., and Ménard, C. (1990). Unsteady flow past a rotating circular cylinder at Reynolds numbers $10^{3}$ and $10^{4}$. J. Fluid Mech. 220, 459-484. doi:10.1017/S0022112090003342

Badr, H. M., and Dennis, S. C. R. (1985). Time dependent viscous flow past an impulsively started rotating and translating circular cylinder. J. Fluid Mech. 158, 447-488. doi:10.1017/S0022112085002725

Biswas, G., and Sarkar, S. (2009). Effect of thermal buoyancy on vortex shedding past a circular cylinder in cross-flow at low Reynolds numbers. Int. J. Heat Mass Transf. 52, 1897-1912. doi:10.1016/j.ijheatmasstransfer.2008.08.034

Cheng, C.-Y. (2006). The effect of temperature-dependent viscosity on the natural convection heat transfer from a horizontal isothermal cylinder of elliptic cross section. Int. Commun. Heat Mass Transf. 33, 1021-1028. doi:10.1016/j. icheatmasstransfer.2005.10.012

Chhabra, R. P., Soares, A. A., Ferreira, J. M., and Caramelo, L. (2007). Effects of viscous dissipation on heat transfer between an array of long circular cylinders and power law fluids. Can. J. Chem. Eng. 85, 808-816. doi:10.1002/cjce.5450850602

Dennis, S. C. R., and Chang, G.-Z. (1970). Numerical solutions for steady flow past a circular cylinder at Reynolds numbers up to 100. J. Fluid Mech. 42, 471-489. doi:10.1017/S0022112070001428

Fornberg, B. (1980). A numerical study of steady viscous flow past a circular cylinder. J. Fluid Mech. 98, 819-855. doi:10.1017/S0022112080000419

Gebhart, B. (1962). Effects of viscous dissipation in natural convection. J. Fluid Mech. 14, 225-232. doi:10.1017/S0022112062001196

Juncu, G. (2004). Unsteady conjugate heat/mass transfer from a circular cylinder in laminar crossflow at low Reynolds numbers. Int. J. Heat Mass Transf. 47, 2469-2480. doi:10.1016/j.ijheatmasstransfer.2003.10.035 types of flow configurations, namely, parallel and contra flows have been considered depending on the directions of the forced convection and the free convection. The dimensionless equations are solved using finite-difference method. For both parallel and contra flows, the Nusselt number and the isotherms strongly depend on the viscous dissipation. The vorticity distribution of the contra flow is relatively more dependent on the viscous dissipation compared to the parallel flow. In contrast to the parallel flow, the point of separation occurs quickly, and the size of the vortex is large for contra flow under the same boundary conditions.

\section{AUTHOR CONTRIBUTIONS}

The development of the model and code has been done by $\mathrm{MH}$. Computations of the model equations have been accomplished by NR. AA-R kept his contribution by adding idea on the discussion from the obtained results jointly with $\mathrm{MH}$.

Juncu, G. (2008). Unsteady heat transfer from an elliptic cylinder. Int. J. Heat Mass Transf. 51, 920-928. doi:10.1016/j.ijheatmasstransfer.2007.11.001

Kurdyumov, V. N., and Fernández, E. (1998). Heat transfer from a circular cylinder at low Reynolds numbers. J. Heat Transfer 120, 72-76. doi:10.1115/1. 2830067

Mahfouz, F. M. (2003). Transient free convection from a horizontal cylinder placed in a micropolar fluid. Heat Mass Transf. 39, 455-462. doi:10.1007/s00231-0020329-4

Mahfouz, F. M., and Badr, H. M. (2000). Flow structure in the wake of a rotationally oscillating cylinder. J. Fluids Eng. 122, 290-301. doi:10.1115/1.483257

Nakai, S., and Okazaki, T. (1975). Heat transfer from a horizontal circular wire at small Reynolds and Grashof numbers-II. Int. J. Heat Mass Transf. 18, 397-413. doi:10.1016/0017-9310(75)90029-0

Nguyen, H. D., Paik, S., and Douglass, W. (1996). Unsteady mixed convection about a rotating circular cylinder with small fluctuations in the free-stream velocity. Int. J. Heat Mass Transf. 39, 511-525. doi:10.1016/0017-9310(95)00149-4

Roache, P. J. (1998). Computational Fluid Dynamics, 2nd Edn. Albuquerque: Hermosa.

Sarkar, S., Dalal, A., and Biswas, G. (2010). Mixed convective heat transfer from two identical square cylinders in cross flow at $\mathrm{Re}=100$. Int. J. Heat Mass Transf. 53, 2628-2642. doi:10.1016/j.ijheatmasstransfer.2010.02.053

Sarkar, S., Dalal, A., and Biswas, G. (2011). Unsteady wake dynamics and heat transfer in forced and mixed convection past a circular cylinder in cross flow for high Prandtl numbers. Int. J. Heat Mass Transf. 54, 3536-3551. doi:10.1016/ j.ijheatmasstransfer.2011.03.032

Conflict of Interest Statement: The authors declare that the research was conducted in the absence of any commercial or financial relationships that could be construed as a potential conflict of interest.

Copyright (C) 2017 Al-Rashed, Roy and Hossain. This is an open-access article distributed under the terms of the Creative Commons Attribution License (CC $B Y)$. The use, distribution or reproduction in other forums is permitted, provided the original author(s) or licensor are credited and that the original publication in this journal is cited, in accordance with accepted academic practice. No use, distribution or reproduction is permitted which does not comply with these terms. 